\title{
Research on The application of Computer Network Communication System on Fire Safety Work
}

\author{
Huaiyi Li \\ Public security fire force academy, Yunnan province 650032, China
}

Key words: computer network communication, fire safety, fire work.

\begin{abstract}
In this article, firstly, the role of computer network communication system in fire safety work is analyzed from two aspects of application principle and different application places. Based on that, this paper focuses on the common problems in the application of computer network communication system in fire protection work, and puts forward corresponding countermeasures against these problems, then, the future direction of the application of computer network communication system in fire protection is discussed.
\end{abstract}

\section{Application of Computer Network Communication System In Fire Safety Work}

\subsection{Application Principle}

Through the establishment of fire safety network system, computer network communication system has significantly improved the efficiency of information transmission, to reflect the accuracy of the information, the information can be real-time monitoring in the fire safety system, if it is found that there is fire hazards, in a timely manner to the computer network transmission control, and alarm. If administrators find fire hazards, they have to taking measures immediately. The second principle is the information security in open network environment, in order to strengthen the control of the virus hidden under the network environment, fire information transmission must grasp this principle, once the virus threat to the information in the transmission or in use, the database paralysis may occur, subsequent fire protection work is also very difficult to carry out. We must ensure safety to work smoothly in the next [1]. In some fire prevention key units, to establish independent fire control network center with the computer network communication system to carry out fire safety work, we can determine the independent operation of the network system, data transmission interference can be reduced to a minimum. The data information can be managed by the backup method. Even if the information loss occurs in the network environment, the recovery of the backup data can also ensure that the system function is not affected.

\subsection{The Application of Computer Network Communication System In Specific Places}

\subsubsection{Crowded Public Places}

Because of the people flow more in public places, the fire safety work also increased the difficulty, in order to achieve a more accurate control of supervision in a timely manner, through the application of computer network system, we can realize the communication between the scene and the total control center. The implementation of the system is not affected by distance, and the speed of fire dispatching is also improved. Once a crowded place fired, it is easy to cause casualties or even an explosion. The earlier the measures are taken, the better the disposal effect in the fire accident, Prior safety control must be strengthened to minimize the probability of fire occurrence, Evacuation of the people in public places is the first step in fire control, and the protection of the lives and property of the masses is the essential content of fire protection work. And the computer network communication system can also realize automatic supervision and control, even if the Employees in public places have been off duty, a small number of people can also control the fire safety system quickly [2].

\subsubsection{Family and Small Public Places}

Fire safety management in small-scale areas need to show higher sensitivity. Our family fire safety system has the advantages of simple operation, easy to understand the secrets of fire tips, and control area can be more precise. Restricted by environment and condition, the automated network control is 
not possible in small locations, Computer network communication system is only a small part of the application, this application more emphasis on data storage and alarm function. Smoke alarms are our most frequently used, when a fire occurs in a small public place, such as a residential building, when the sensor has sensed the smoke, the system transmit the alarm signal to the general control center, After the control center staff finds out the problem, the system can organize evacuation and control the fire immediately. The information transmission function of computer network communication system has played an important role in it [3]. transmission function of computer network communication system has played an important role in it [3].

\subsubsection{Flammable and Explosive Chemical Plants, Large Storage Tank Area}

Once such a place fires, it is easy to cause an explosion. It is necessary to establish an automatic fire extinguisher through the computer network communication system, when the alarm is made, the fire extinguishing device is automatically started to control the fire sufficiently, so as to avoid an explosion. When a fire occurs in a production environment in a chemical plant, the communication system also automatically stops production lines through instructions, by blocking the flammable gas, to avoid the scope of fire continues to expand [4]. We can automatically design functions of the system in a computer network environment, can start with the common fire types and strengthen the prevention and control, give full play towards the advanced, convenient and high efficiency of network system, through the method of combining software and hardware, a complete fire safety control system is constructed. The most important characteristic of the application of network technology is the liberation of staff, complex supervision and control tasks can be accomplished without the need for more staff.

\section{Common Problems And Solutions In Computer Network Communication System}

\subsection{Fake Alarm Problem}

\subsubsection{The Cause of the Problem}

This problem is mainly caused by the error in the transmission of information, the error message that is not in conformity with the actual situation, and which cannot reflect the real situation in the transmission process, and will eventually affect the final information security. The sensitivity of detector is the key factor affecting the quality of information transmission, smoking is usually forbidden in the fire control area, Smoke generated by smoking can easily cause false fire alarms. When the smoke is very large, the results detected by the temperature sensing device are prone to errors, after the wrong information is transmitted to the general control center, the fire extinguishing system starts automatically. The interference information received by sensors is also the main cause of this problem, the jamming information is then transmitted to the control center, when the control center receives the instruction, it will make a wrong response, and it cannot help solve the current problems

\subsubsection{Prevention and Control Measures for This Problem}

In order to improve the stability of the final control results, we can strengthen the security control of the information transmission system, and regularly implement the maintenance of the system. If the detection sensors in public place are not maintained for a long time, the dust and garbage on the surface will be more and more, regularly clean up these things, the sensitivity of the sensor can be resumed, and we could adjust the site control through this method. Only if the security transmission of information about the control center is guaranteed, the follow-up control plan will not be affected. The detection device is the first defending line of the computer network communication system used on fire safety, only by ensuring the accuracy of the results of the transmitted information, and then, none of the tasks will be affected, so as to achieve the desired effect of control management. Technical person tested the information system in their daily work, they should also strengthen professional knowledge training to make sure that when they are finding information error and other problems, it can be solved the first time. These measures are used to avoid Fake alarms from the root and then to improve the system's efficiency . 


\subsection{Omit Alerts Problem}

\subsubsection{The Cause of the Problem}

This problem is mainly caused by the failure of network information system, in the process of information transmission updating, there is a phenomenon of network connection interruption, after the re-connection, some part of the information is lost, this information is affected by the network and some of this information are not transmitted to the control system, it is easy to cause the omitting fire alerts. The application of computer network technology requires a complete process of construction, if the system is Immature, it will easily cause this problem. There is no guarantee of safety in the process of information transmission, In most cases, the network communication system used in the fire safety work is connected to the local area network, and other devices are not allowed to be inserted into computer control devices. Some fire safety control person operating irregularities in the work, they connect personal devices to the control computer, the virus has the opportunity to invade, and eventually lead to damage to the computer's internal information, this resulting in serious failure to reporting fire alarm, fire accident cannot be found at once. Because of the reasons above, Omitting alerts problem has finally occurred

\subsubsection{Prevention and Control Measures for This Problem}

For fire safety control, we should learn to use effective prevention and control methods to prevent some non working equipment from being connected to the system, and regularly check the system for viruses, observe whether there are hidden dangers of the virus, and to solve them. Strengthen the combination of software, control systems and hardware, we can test the consistency of the data after the design was completed, if information is not missing or there is no transmission error occurs, the system can be put into use. We must also strengthen the control and management of software systems to ensure that software operations are not disturbed by other factors, only in this way, can we have to making the information transmission system to achieve the best results. Fire safety control is a highly accurate work, any error problems may affect the safety of human life and property, therefore, we must strengthen the adjustment control at the site, and regularly detect safety, so, problems that may arise in accuracy can also be addressed at the root. Controllers, sensors, and transmission lines form a robust system. Such design will not cause information loss in the transmission of information.

\subsection{Preventive Measures For High Rate Of System Error Reporting}

The smoke detector and the temperature sensor can be combined to solve the fake fire alarm problem, only both two detectors send warning messages to confirm that this is an effective fire alarm signal. By adopting an intelligent computer system, the program is setting up two times to confirm, only both two detectors are issued an alarm before it can be confirmed as a formal alarm, Of course, one detector alarm should also be marked.

\section{Virtual Reality Technology Based On Computer Network Communication System}

Virtual reality is the latest research and development technology in recent years, the real scene can be simulated by simulation technology, which is most often used in fire safety drills, the sensory effects created in this way can be very close to reality. In the traditional fire safety drills, they are usually just performing evacuation simulation exercises, did not create a simulation scene, In the event of a real fire, not only will it cause panic among the masses, but also there may still be difficulties in the process of safe evacuation [5]. Virtual technology is constructed with the help of computer network platform, which can realize remote security training, enhance actual combat experience, and improve fire evacuation efficiency. We can use virtual reality technology to carry out fire drills to improve the efficiency of fire control, and actively carry out prevention and control work, at the same time, in the simulation exercise, we can detect which functions as the fire safety information system need to be optimized and perfected, through optimization and improvement, the use of database can be further improved, and the information about the regulatory control area can be updated in real-time [6]. 
In the future, computer network communication systems will develop in the direction of artificial intelligence, we can combine artificial intelligence with automation to form an imitation expert system, the prevention and control of fire from this system can also be carried out from the expert level. It is more in line with the actual workflow, and also can achieve more efficient control effect, this system also has memory function, in order to avoid the scope of fire continues to expand, threatening the safety of people's lives and property, this system can fully understand the fire scene and adjust automatically according to the received data. At the same time, the corresponding command is issued to control the fire. In the application of computer network communication system, in order to avoid idle functions and waste of information resources, functional selection should be carried out according to the actual situation, choosing the appropriate network communication system can help improving the efficiency of supervision and control, and also more in line with the actual needs of the field.

\section{Conclusion}

With the combination of computer control technology and network communication system, the mode of fire safety work has been changed obviously, and the working methods are also more diversified. The concept that a single computer serves all of the computer's needs in the organization is quickly replaced by a large number of distributed but interconnected computers that are implemented together. Such system is called computer network. In the long run, using the Internet to enhance interpersonal communication may be more important than its technical purpose (such as increased reliability). Through the computer network, we integrate the automatic fire alarm system with the fire command and dispatch system, which can be connected through copper wire, the optical fiber, microwave or communication satellites. Through the establishment of interconnected fire automatic alarm network scheduling monitoring system, we can timely and accurately forecast the fire, activate the fire extinguishing system and related fire extinguishing equipment in time, and minimize the fire loss.

\section{Reference}

[1] Li Hongshan, Liu Zhe. The application of computer network and communication system for fire prevention [J]. Journal of the armed police academy, 2009 (4): 71-72

[2] anonymous. "13th Five-Year" national key researched project "social issues of fire safety management and firefighting and rescue information to support key technology" will be held to start construction standardization [J]., 2016 (11): 86-86.

[3] Tian Xiangxia. Discussion on the hidden danger and prevention strategy of computer network for public security fire control [J]. information security and technology, 2011 (8): 31-32.

[4] Liu Lizhen, Chen Lei, Zhang Maohong. A fire safety assessment model on large urban communities based on Bayesian networks [J]. computer application research, 2011,28 (1): 182-184.

[5] Zhang Lei, Zhu Guoqing, Guo Dagang. Research on fire evacuation training system for public buildings based on VR [J]. Fire science and technology, 2015 (4): 526-529.

[6] Gong Zheng. Application of VR technology in daily training of fire control [J]. Jiangxi chemical industry, 2017 (2): 214-215. 